\title{
INVENTARIO RÁPIDO DE LAS ARAÑAS (ARACHNIDA: ARANEAE) DEL REFUGIO DE VIDA SILVESTRE EL CAÑÓN DEL RÍO GURABO, REPÚBLICA DOMINICANA
}

\section{Rapid inventory of the spiders (Arachnida: Araneae) from the wildlife refuge El cañón del río Gurabo, Dominican Republic}

\author{
Gabriel de los Santos y Solanlly Carrero Jiménez
}

\begin{abstract}
Museo Nacional de Historia Natural "Prof. Eugenio de Jesús Marcano". C/ César Nicolás Penson, Plaza de la Cultura Juan Pablo Duarte, Santo Domingo, 10204, República Dominicana. g.delossantos@mnhn.gov.do, s.carrero@mnhn.gov.do.
\end{abstract}

\section{RESUMEN}

Se ofrecen los resultados de un inventario de las arañas del refugio de vida silvestre El cañón del río Gurabo, República Dominicana. Se registran 73 especies, 64 géneros y 32 familias para esta área protegida. Se amplía la distribución conocida de Yabisi guaba Rheims et Brescovit, 2004 (Hersiliidae), una especie endémica rara. Se comenta la distribución de las especies endémicas Antillena sp. y Pozonia sp., y la especie exótica invasora Cyrtophora citricola (Forskål, 1775). Se consideran como objetos de conservación del área protegida las siguientes 16 especies endémicas: Anasaitis elegantissima (Simon, 1888), Anelosimus fraternus Bryant, 1948, Antillena sp., Ariadna sp., Bolostromus sp., Cyrtopholis sp., Hentzia mandibularis (Bryant, 1943), Jollas crassus (Bryant, 1943), Modisimus cuadro Huber et Fischer, 2010, Modisimus sp., Odo sp., Pozonia sp., Selenops sp., Trichopelma sp., Truncattus sp., Yabisi guaba Rheims et Brescovit, 2004.

Palabras clave: inventario rápido, diversidad, distribución, Hispaniola.

\section{ABSTRACT}

The results of an inventory of the spiders from El cañón del río Gurabo wildlife refuge, Dominican Republic are presented. A total of 73 species, 64 genera and 32 families are registered from this protected area. The known distribution of Yabisi guaba Rheims et Brescovit, 2004 (Hersiilidae), a rare endemic species, is extended. The distribution of the endemic species Antillena sp. and Pozonia sp., as well as the exotic invasive species Cyrtophora citricola (Forskål, 1775), is commented. The following 16 endemic species are considered as conservation targets for this refuge: Anasaitis elegantissima (Simon, 1888), Anelosimus fraternus Bryant, 1948, Antillena sp., Ariadna sp., Bolostromus sp., Cyrtopholis sp., Hentzia mandibularis (Bryant, 1943), Jollas crassus (Bryant, 1943), Modisimus cuadro Huber et Fischer, 2010, Modisimus sp., Odo sp., Pozonia sp., Selenops sp., Trichopelma sp., Truncattus sp., Yabisi guaba Rheims et Brescovit, 2004.

Keywords: rapid inventory, diversity, distribution, Hispaniola.

El refugio de vida silvestre El cañón del río Gurabo abarca territorios de las secciones Los Quemados, Las Caobas, Gurabo y El Cacique, la primera de las cuales pertenece a la provincia Valverde, en tanto que las demás corresponden a la provincia Santiago Rodríguez 
(MIMARENA, 2018). Esta área protegida se encuentra ubicada entre las coordenadas $19^{\circ} 26^{\prime} 60^{\prime \prime}$ $\mathrm{N}$ a $19^{\circ} 19^{\prime} 60^{\prime \prime} \mathrm{N}$ y $71^{\circ} 10^{\prime} 00^{\prime \prime} \mathrm{O}$ a $71^{\circ} 12^{\prime} 00^{\prime}$ ' O, con una extensión de $30.16 \mathrm{~km}^{2}$ y elevaciones entre 60 y $250 \mathrm{~m} \mathrm{~s}$. n. m. La vegetación en esta zona consiste en bosque seco, matorral seco, bosque latifoliado húmedo y pastizal, así como una vegetación ribereña asociada al río Gurabo y sus afluentes (MIMARENA, 2018).

Los trabajos faunísticos en este refugio han incluido tres grupos de vertebrados: aves, anfibios y reptiles (Landestoy y Ortiz, 2015; Ortiz y Landestoy, 2015; MIMARENA, 2018), faltando información sobre otros grupos, particularmente de arácnidos.

Con el objetivo de aumentar el conocimiento sobre la diversidad de la fauna de este refugio, recientemente investigadores del Museo Nacional de Historia Natural "Prof. Eugenio de Jesús Marcano" realizaron recientemente dos expediciones a esta área protegida. Estas expediciones tuvieron lugar del 29 de mayo al 1ro de junio y del 11 al 14 de septiembre de 2018. Además de las arañas, fueron estudiados los reptiles (Gabot-Rodríguez y Marte, 2019), anfibios, murciélagos, peces, insectos y miriápodos, al igual que otros arácnidos (resultados aún sin publicar).

Se realizaron colectas libres diurnas (09:00 - 15:00 horas) y nocturnas (20:00 - 00:00 horas). Estas colectas tuvieron lugar en los bosques seco y húmedo. Los especímenes se introdujeron en alcohol etílico al $70 \%$ y se depositaron en la colección de arácnidos del museo (MNHNSD).

Durante el inventario fueron reconocidas 73 especies de arañas (Anexo 1), las cuales representan el $16.9 \%$ de las especies conocidas para la Hispaniola (República Dominicana y Haití); se identificaron 32 familias y 64 géneros. De las especies registradas, 16 son endémicas de la Hispaniola, representando el $7.1 \%$ de las especies endémicas de la isla.

El presente trabajo representa el primer registro de las especies listadas para esta área específica, destacándose Antillena sp. (Theraphosidae; Fig. 1), Pozonia sp. (Araneidae) y Yabisi guaba Rheims et Brescovit, 2004 (Hersiliidae). Del género monotípico y endémico de la Hispaniola Antillena Bertani, Huff et Fukushima, 2017, se conocen registros de las provincias Pedernales, Independencia y Barahona (Bertani y Huff, 2013; de los Santos y Carrero Jiménez, 2015; Kaderka, 2016; Fukushima y Bertani, 2017), por lo que ahora se registra para el norte de República Dominicana. Asimismo, la especie Pozonia sp. representa el primer registro de este género para el norte de la República Dominicana; los registros previos, todos correspondientes a la especie endémica Pozonia andujari Alayón, 2007, provienen de las provincias Peravia, San Juan, San José de Ocoa y La Altagracia (Alayón García, 2007; Alayón García et al., 2011). Por otro lado, Y. guaba es una especie endémica, muy rara, de la que solo se han colectado tres ejemplares, en las provincias Pedernales y Montecristi (Rheims y Brescovit, 2004; Rheims et al., 2014), ampliándose su distribución conocida con el actual registro.

De igual forma, también se extiende la distribución conocida de la "araña parda del Mediterráneo" (Cyrtophora citricola), especie exótica invasora, de la que se tenían registros en las provincias Bahoruco, Barahona, Independencia, Pedernales, San Juan, Peravia, La Vega, Santo Domingo, La Romana, Hato Mayor y La Altagracia (Alayón García et al., 2001; Teruel et al., 2014; de los Santos y Carrero Jiménez, 2015); además de estos registros, la colección del MNHNSD guarda ejemplares colectados en las provincias Azua, San Cristóbal, Monseñor Nouel y Montecristi. 


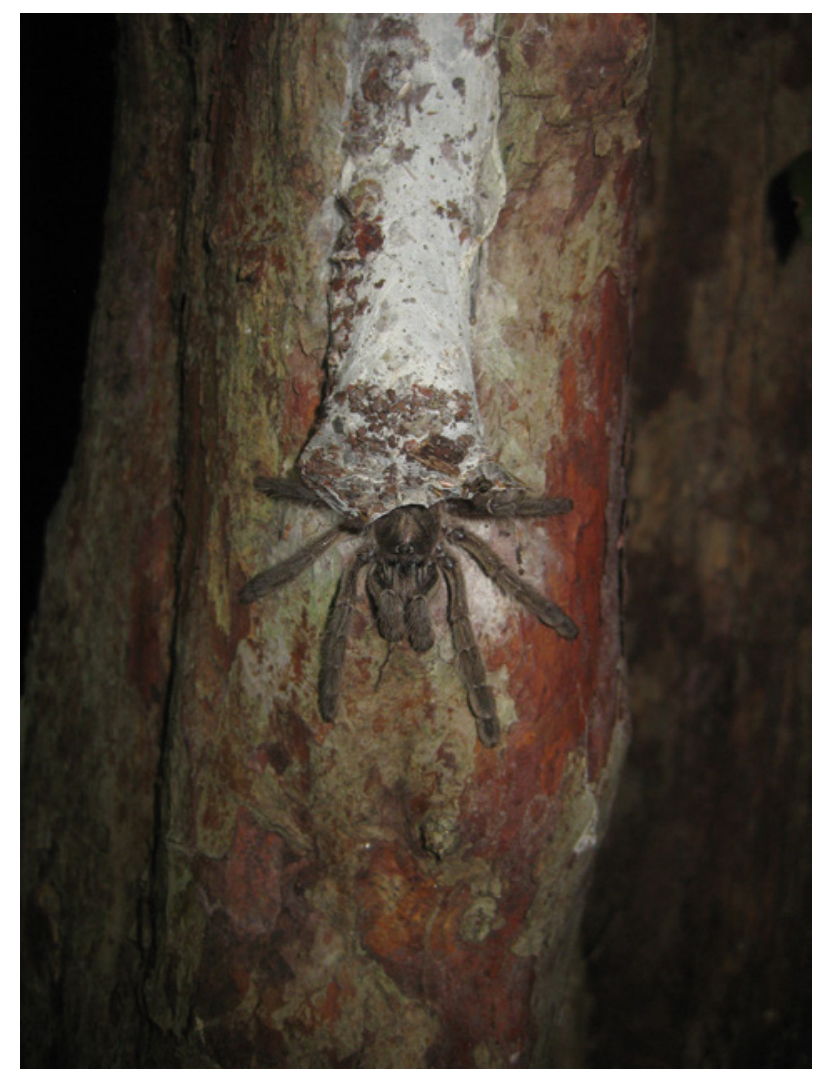

Figura 1. Hembra de Antillena sp. (Theraphosidae). Ejemplar de Antillena sp. en su típica madriguera.

Como objetos de conservación del refugio de vida silvestre Cañón del río Gurabo, se eligieron las especies endémicas siguientes: Anasaitis elegantissima (Simon, 1888), Anelosimus fraternus Bryant, 1948, Hentzia mandibularis (Bryant, 1943), Jollas crassus (Bryant, 1943), Modisimus cuadro Huber et Fischer, 2010 y Yabisi guaba Rheims et Brescovit, 2004. A éstas se suman otras 10 especies aún sin determinar, las que pasan a estar en proceso de descripción como nuevas especies: Antillena sp., Ariadna sp., Bolostromus sp., Cyrtopholis sp., Modisimus sp., Odo sp., Pozonia sp., Selenops sp., Trichopelma sp. y Truncattus sp. De validarse estas futuras descripciones, constiuirían endemismos locales.

\section{AGRADECIMIENTOS}

A Ramón Aníbal Almonte y Jonatan Brito, Ministerio de Medio Ambiente y Recursos Naturales (prov. Valverde), por el apoyo logístico dentro y fuera del área protegida. Al equipo del Museo Nacional de Historia Natural "Profesor Eugenio de Jesús Marcano" que nos acompañó en las expediciones y de igual forma a los guardaparques y brigadistas José A. Uceta, Johnny Rodríguez y Rafael Uceta, por su valiosa ayuda en el campo. Al Ministerio de Medio Ambiente y Recursos Naturales, Santo Domingo, por el permiso de investigación otorgado al proyecto "Inventario rápido de fauna en el refugio de vida silvestre Cañón del río Gurabo". A Miguel Landestoy y Antonio Tosto por informarnos, previo a este inventario, sobre la presencia del género Antillena en esta área. 


\section{LITERATURA CITADA}

Alayón García, G. 2007. Especie nueva de Pozonia (Araneae:Araneidae) para República Dominicana. Solenodon, 6: 41-44.

Alayón García, G., G. de los Santos y S. Carrero Jiménez. 2011. Nuevos registros de Pozonia andujari (Araneae: Araneidae) para la República Dominicana. Novitates Caribaea, 4: $134-135$.

Bertani, R. y J. Huff. 2013. Avicularia rickwesti sp. nov., a remarkable new species of Avicularia (Theraphosidae: Aviculariinae) from Dominican Republic. Zoologia, 30 (3): 333-337.

De los Santos, G. y S. Carrero Jiménez. 2015. Inventario de las arañas (Arachnida: Araneae) del Parque Nacional Sierra Martín García, República Dominicana, con nuevos registros de género y especie para la Hispaniola. Novitates Caribaea, 8: 131-137.

Fukushima, C. S. y R. Bertani. 2017. Taxonomic revision and cladistic analysis of Avicularia Lamarck, 1818 (Araneae, Theraphosidae, Aviculariinae) with description of three new aviculariine genera. ZooKeys, 659: 1-185. https://doi.org/10.3897/zookeys.659.10717.

Gabot-Rodríguez, E. y C. Marte. 2019. Inventario de los reptiles del refugio de vida silvestre El cañón del río Gurabo, República Dominicana. Novitates Caribaea, 13: 117-121.

Kaderka, R. 2016. Description of the male of Avicularia rickwesti Bertani \& Huff, 2013, a remarkable species from the Dominican Republic (Araneae: Theraphosidae: Aviculariinae). Revista Ibérica de Aracnología, 28: 121-127.

Landestoy, M. y R. Ortiz. 2015. Listado de los anfibios y reptiles registrados en el Refugio de Vida Silvestre Cañón (Furnia) del Río Gurabo y entorno 2009-2015. 3 pp. [Sin publicar]. Disponible en https://www.rufford.org/files/Checklist $\% 20$ for\%20the $\% 20$ Herpetofauna_0. pdf. Accedido en 12-X-2018.

Ministerio de Medio Ambiente y Recursos Naturales [MIMARENA], Viceministerio de Áreas Protegidas y Biodiversidad, Dirección de Vida Silvestre. Julio de 2018. Plan de manejo, refugio de vida silvestre Furnia de Gurabo. Santo Domingo, República Dominicana, 60 pp. [Sin publicar].

Ortiz, R. A. y M. A. Landestoy T. 2015. Listado de la avifauna registrada en el Refugio de Vida Silvestre Cañón (Furnia) del Río Gurabo y entorno 2009-2015. 4 pp. [Sin publicar]. Disponible en https://www.rufford.org/files/Listado\%20de\%20la\%20Avifauna.pdf. Accedido en 12-X-2018.

Rheims, C. A. y A. D. Brescovit. 2004. Revision and cladistic analysis of the spider family Hersiliidae (Arachnida, Araneae) with emphasis on Neotropical and Nearctic species. Insect Systematics \& Evolution, 35: 189-239.

Rheims, C. A., I. Agnarsson y G. A. Alayón. 2014. Description of the male of Yabisi guaba (Araneae: Eresoidea: Hersiliidae). Zoologia, 31 (4): 405-407. http://dx.doi.org/10.1590/ S1984-46702014000400013.

Teruel, R., Y. Martín-Castejón, F. Cala, G. Alayón García y T. M. Rodríguez-Cabrera. Actualización de la distribución de Cyrtophora citricola (Forskål, 1775) (Araneae: Araneidae) en Cuba y Las Antillas. Revista Ibérica de Aracnología, 25: 27-32. 


\section{LISTA DE ESPECIES DEL REFUGIO DE VIDA SILVESTRE \\ EL CAÑÓN DEL RÍO GURABO}

* = endémica. EX, exótica invasora. Inm., inmaduro. BS, bosque seco; BH, bosque húmedo.

\section{INFRAORDEN MYGALOMORPHAE}

\section{CYRTAUCHENIIDAE}

1. Bolostromus sp.* 4; $\mathrm{BH}$

DIPLURIDAE

2. Masteria sp. 19 ; BH

\section{THERAPHOSIDAE}

3. Antillena sp.* 4 + + ; BS, BH

4. Cyrtopholis sp.*3우; $\mathrm{BH}$

5. Phormictopus cancerides (Latreille, 1806) 2 우 1 ^ 3 inm.; BS, BH

6. Trichopelma sp.* 1 ㅇ 1 inm; BS

\section{INFRAORDEN ARANEOMORPHAE}

\section{AGELENIDAE}

7. Barronopsis sp. 2 ㅇ $2 \hat{\jmath}$; $\mathrm{BS}$

\section{ANYPHAENIDAE}

8. Hibana sp. $1 \delta^{\Uparrow}$ inm. 1 inm.; BH, BS

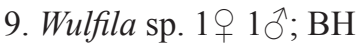

10. Anyphaenidae sp. 4ðे; BH

\section{ARANEIDAE}

11. Acacesia hamata (Hentz, 1847) $10 \hat{~} 1$ inm.; BS

12. Cyrtophora citricola (Forskål, 1775) EX 1\%; BS

13. Eustala anastera (Walckenaer, 1842) 2 ; 4 inm; BS, BH

14. Gasteracantha cancriformis (Linnaeus, 1758) $3 q 1$ inm.; BS

15. Larinia sp. $1 ð$ inm.; BS

16. Mecynogea martiana (Archer, 1958) 19; BS

17. Metazygia crewi (Banks, 1903) 3 q 101 inm.; BH, BS

18. Micrathena forcipata (Thorell, 1859) 39 ; BH, BS

19. Ocrepeira serrallesi (Bryant, 1947) 3 \% ; BH, BS

20. Parawixia tredecimnotata F. O. P.-Cambridge, 19042 + 1 ^ 2 inm.; BH, BS

21. Pozonia sp.*1\%; BS

\section{CAPONIIDAE}

22. Nops sp. 1 ; ; $\mathrm{BH}$

\section{CTENIDAE}

23. Cupiennius sp. 2 inm.; BH

24. Ctenidae sp. 4 inm.; BH 


\section{CORINNIDAE}

25. Corinna sp. 1 q $2 \hat{\diamond} 6$ inm.; BH, BS

26. Mazax sp. 1ð̋; BS

\section{DEINOPIDAE}

27. Deinopis lamia MacLeay, 18391 q $2 \hat{\jmath}$; BH, BS

FILISTATIDAE

28. Filistatidae sp. 2 inm; BH

\section{GNAPHOSIDAE}

29. Camillina sp. 1 ; ; $\mathrm{BH}$

30. Zelotes sp. 1 으 $\mathrm{BS}$

31. Gnaphosidae sp.1 1 ㅇ BS

32. Gnaphosidae sp.2 2\%; BS

HERSILIIDAE

33. Yabisi guaba Rheims et Brescovit, 2004*1우 1ठ; BS

\section{LYCOSIDAE}

34. Trochosa sp. $3 q 1{ }^{\lambda}$; BS, BH

MIMETIDAE

35. Mimetus sp. 1 ; ; BH

\section{MITURGIDAE}

36. Teminius insularis (Lucas, 1857) 1q; BS

\section{OONOPIDAE}

37. Oonopidae sp.1 1 inm.; BH

38. Oonopidae sp. 2 1ठ; BH

\section{PHOLCIDAE}

39. Micropholcus sp. 1q; BH

40. Modisimus cuadro Huber et Fischer, 2010*1우 BH

41. Modisimus sp.* 1 §’; $\mathrm{BH}$

PISAURIDAE

42. Thaumasia velox Simon, 18981 ㅇ 1 inm.; BH

\section{SALTICIDAE}

43. Agobardus sp. 1 \% $\mathrm{BH}$

44. Anasaitis elegantissima (Simon, 1888)* 1 ふ̋; BS

45. Anasaitis sp. 3 ㅇ 1ふ; BS, BH

46. Hentzia mandibularis (Bryant, 1943)*1ð’; BS

47. Jollas crassus (Bryant, 1943)* 2 ○ 1ठ; BS, BH

48. Lyssomanes antillanus Peckham, Peckham et Wheeler, $18891{ }^{\Uparrow} 1$ inm.; BH

49. Truncattus sp.*1\%; BS 


\section{SCYTODIDAE}

50. Scytodes fusca Walckenaer, 18373 \% ; BS

51. Scytodes longipes Lucas, 1844 4; BS, BH

\section{SEGESTRIIDAE}

52. Ariadna sp.* 4\%; BH

\section{SELENOPIDAE}

53. Selenops sp.* $1{ }^{\Uparrow}$; BS

\section{SICARIIDAE}

54. Loxosceles sp. 3 q inm.; BS

\section{SPARASSIDAE}

55. Neostasina sp. 1 đ inm. 3 inm.; BS, BH

\section{TETRAGNATHIDAE}

56. Alcimosphenus licinus Simon, 1895 1; BH

57. Leucauge argyra (Walckenaer, 1842) 1q; BS

58. Tetragnatha cf. elongata Walckenaer, 18411 우 $1 \overbrace{}^{\lambda}$; BH

\section{THERIDIIDAE}

59. Anelosimus fraternus Bryant, 1948*2\%; BH

60. Dipoena sp. 1 ; ; BH

61. Faiditus caudatus (Taczanowski, 1874) 19; BH

62. Latrodectus geometricus C. L. Koch, 18412 우숭 BS, BH

63. Neopisinus gratiosus (Bryant, 1940) 39 ; BH

64. Neospintharus fuscatus (O.P.-Cambridge, 1894) 1ठ઼; BS

65. Pholcomma sp. 1 đ̄ inm; $\mathrm{BH}$

66. Steatoda erigoniformis (O.P.-Cambridge, 1872) 19; BH

67. Tidarren sisyphoides (Walckenaer, 1842) 19; BH

68. Theridion sp. $1 \lesssim$; $\mathrm{BH}$

THERIDIOSOMATHIDAE

69. Wendilgarda sp. $1{ }^{\jmath}$; $\mathrm{BH}$

\section{THOMISIDAE}

70. Isaloides sp. $3 \circlearrowleft$ inm.; $\mathrm{BH}$

TRACHELIDAE

71. Trachelas sp. $2 \widehat{\jmath}$; $\mathrm{BH}$

\section{ULOBORIDAE}

72. Miagrammopes sp. 2 + 1 inm.; BH, BS

\section{XENOCTENIDAE}

73. Odo sp.* 1 ㅇ $1 \delta^{\lambda}$ inm.; BS 